\title{
Discontinuation and restarting in patients on statin treatment: prospective open cohort study using a primary care database
}

\author{
Yana Vinogradova, ${ }^{1}$ Carol Coupland, ${ }^{1}$ Peter Brindle, ${ }^{2,3}$ Julia Hippisley-Cox ${ }^{1}$
}

1Division of Primary Care,

University of Nottingham,

University Park, Nottingham,

NG2 7RD, UK

${ }^{2}$ Avon Primary Care Research

Collaborative, Bristol Clinical

Commissioning Group,

Bristol, UK

${ }^{3}$ University of Bristol, Bristol, UK

Correspondence to:

Y Vinogradova

Yana.Vinogradova@

nottingham.ac.uk

Additional material is published online only. To view please visit

the journal online.

Cite this as: BMJ 2016;353:i3305 http://dx.doi.org/10.1136/bmj.i3305

Accepted: 11 May 2016

\begin{abstract}
OBJECTIVES

To estimate rates of discontinuation and restarting of statins, and to identify patient characteristics associated with either discontinuation or restarting.

DESIGN

Prospective open cohort study.

SETTING

664 general practices contributing to the Clinical Practice Research Datalink in the United Kingdom. Data extracted in October 2014.
\end{abstract}

\section{PARTICIPANTS}

Incident statin users aged 25-84 years identified between January 2002 and September 2013. Patients with statin prescriptions divided into two groups: primary prevention and secondary prevention (those already diagnosed with cardiovascular disease). Patients with statin prescriptions in the 12 months before study entry were excluded.

\section{MAIN OUTCOME MEASURES}

Discontinuation of statin treatment (first 90 day gap after the estimated end date of a statin prescription), and restarting statin treatment for those who discontinued (defined as any subsequent prescription between discontinuation and study end).

RESULTS

Of 431023 patients prescribed statins as primary prevention with a median follow-up time of 137 weeks, $47 \%(n=204622)$ discontinued treatment and $72 \%$

\section{WHAT IS ALREADY KNOWN ON THIS TOPIC}

With regards to statin treatment, previous studies have shown relatively high levels of discontinuation or low levels of adherence

Several studies found that younger and older patients, women, ethnic minorities, smokers, patients with lower body mass index, and patients without hypertension or diabetes have lower adherence to statin treatment

However, most studies were not representative of the general population and were inconsistent overall in terms of study design, definitions of exposure, and outcome

\section{WHAT THIS STUDY ADDS}

In this large population based study, ethnic minority status, smoking, and type 1 diabetes were more associated with discontinuation; but among patients who had discontinued, these factors were more associated with restarting, suggesting poor adherence to statin treatment

Type 2 diabetes and hypertension were less associated with discontinuation; among patients who had discontinued, these factors were more associated with restarting, suggesting good adherence to statin treatment

However, younger and older ages, female sex, and liver disease were more associated with discontinuation; among patients who had discontinued, these factors were less associated with restarting-suggesting true discontinuation with statin treatment (or “real stopping”)
( $n=147305)$ of those who discontinued restarted. Of 139314 patients prescribed statins as secondary prevention with median follow-up time of 182 weeks, $41 \%(n=57791)$ discontinued treatment and $75 \%$ ( 43211 ) of those who discontinued restarted. Younger patients (aged $\leq 50$ years), older patients ( $\geq 75$ years), women, and patients with chronic liver disease were more likely to discontinue statins and less likely to restart. However, patients in ethnic minority groups, current smokers, and patients with type 1 diabetes were more likely to discontinue treatment but then were more likely to restart, whereas patients with hypertension and type 2 diabetes were less likely to discontinue treatment and more likely to restart if they did discontinue. These results were mainly consistent in the primary prevention and secondary prevention groups.

\section{CONCLUSIONS}

Although a large proportion of statin users discontinue, many of them restart. For many patient groups previously considered as "stoppers," the problem of statin treatment "stopping" could be part of the wider issue of poor adherence. Identification of patient groups associated with completely stopping or stop-starting behaviour has positive implications for patients and doctors as well as suggesting areas for future research.

\section{Introduction}

There has been a marked increase in statin prescribing over the past 10 years, both for patients with cardiovascular disease and for those at high risk of developing it. ${ }^{1}$ In the United States ${ }^{2}$ and United Kingdom, ${ }^{3}$ recent guidelines have lowered the threshold for consideration of statins in patients without cardiovascular disease from a $20 \%$ risk of developing cardiovascular disease at 10 years $^{4}$ to a $10 \%$ risk at 10 years. ${ }^{23}$ These changes were based on recommendations from the Cholesterol Treatment Trialists meta-analysis $(2012)^{5}$ and a revised Cochrane review (2013). ${ }^{6}$

Concerns have been raised about the balance of harms and benefits of statins, especially for the larger number of statin users with lower absolute risk of cardiovascular disease. ${ }^{7-9}$ Although observational studies have examined the pattern of uptake of statins ${ }^{1011}$ and of unintended effects from statin use, ${ }^{12-14}$ it is unclear which patient characteristics affect discontinuation, in what ways, and by how much. Papers have examined risks of non-adherence and discontinuation, but study designs were inconsistent in terms of patient selection, definition of discontinuation, and availability of possible risk predictors, thus creating challenges for meta-analyses. ${ }^{15} 16$ Results from a few investigations of restarting suggested that most discontinuers eventually 
restarted, but these studies were all based on data only up to $2008 .^{17-19}$ One study investigated factors associated with restarting, but had only a limited number of factors available and was not representative of a general population. ${ }^{18}$

We have, therefore, undertaken a study using a large primary care database in the UK to determine rates of discontinuation and restarting in statin treatment, and to identify those patient characteristics associated with discontinuation and those associated with restarting.

\section{Methods}

Study design

This study design has been fully described elsewhere. ${ }^{20}$ In summary, in October 2014, we extracted information from 664 UK general practices contributing to Clinical Practice Research Datalink (CPRD), identifying an open cohort of patients aged 25-84 years who started statin treatment (the entry date) between 1 January 2002 and 30 September 2013. Patients were excluded if they had less than one year of medical records before or after the entry date, or had statin prescriptions in the 12 months before entering the study. Patients having only one statin prescription during the study period were excluded from the main cohort, being regarded as non-users of statins in the main analyses. Patients were censored if they died or left the practice. Patients without cardiovascular disease at the entry date formed a primary prevention group. If any patients developed cardiovascular disease, they left the study at the date of their diagnosis. Patients diagnosed with cardiovascular disease before or at the entry date formed a secondary prevention group.

\section{Outcomes}

The discontinuation outcome was defined as the first 90 day gap after the estimated end date of a statin prescription, with outcome date as the estimated end date. This 90 day exposure window has been used in previous studies based on routinely collected data in primary care. $^{1221}$ To identify patients who restarted statins after temporarily stopping, we ran a second analysis on those who had discontinued. The restarting outcome was defined as the first statin prescription found after the period of discontinuation, with outcome date as the date of this prescription.

\section{Exposure and covariates}

Five statins commonly prescribed in the UK were considered: simvastatin, atorvastatin, fluvastatin, rosuvastatin, and pravastatin. Daily dose was expressed in potency units to exceed 30\% reduction in low density lipoprotein levels (20 mg of simvastatin, $10 \mathrm{mg}$ of atorvastatin, $80 \mathrm{mg}$ of fluvastatin, $5 \mathrm{mg}$ of rosuvastatin, and $40 \mathrm{mg}$ of pravastatin). ${ }^{22}$ The dose was further categorised as less than one potency unit, one unit, two units, and three or more units. We calculated cumulative duration of exposure as the period between the first prescription date and the estimated end of the final prescription.
To characterise patients who discontinued, did not discontinue, or restarted the treatment, we used several factors:

- Demographics (age, sex, ethnicity, and socioeconomic status)

- Clinical values (smoking status, body mass index, systolic blood pressure, total cholesterol:high density lipoprotein ratio)

- Chronic diseases (rheumatoid arthritis, chronic renal disease, liver disease, atrial fibrillation, treated hypertension, cancer, heart failure, diabetes and dementia)

- Genetic characteristics (family history of premature coronary heart disease and diagnosis of familial hypercholesterolaemia)

- Use of other treatments.

Medication factors included specific drugs related to cardiovascular treatment (aspirin and anticoagulants), and the number of other treatments with systemic effect (tablets or injections) associated with chapters in the British National Formulary. ${ }^{23}$ The description of patients in the primary prevention group also included their 10 year cardiovascular risk calculated by the QRISK2-2014 score.

Records for covariates were considered if they were recorded before or at the entry date. To achieve more complete records, we extracted ethnicity information from both the CPRD and the link to Hospital Episode Statistics. Deprivation was measured by the Townsend score (divided into five equal groups). Scores based on the patient's residential area were available for only $62 \%$ of patients; therefore, for the main analysis, we used the general practice area deprivation scores. In an additional analysis on this subgroup of $62 \%$, we used patient based scores.

\section{Statistical analysis}

The analysis was run separately for the primary and secondary prevention groups. We calculated the incidence of discontinuation during follow-up by dividing the number of incident stoppers by the number of person years. Patients were considered long term users rather than short term users if they used statins for more than a year. Discontinuation was pictorially described by Kaplan-Meier curves.

To investigate the associations between the covariates at study entry and the risk of statin discontinuation, we used multivariate Cox regression analysis. Age, sex, ethnic group, practice based deprivation group, calendar year, type and dose of statin at the study entry, chronic diseases, clinical values, genetic characteristics, and use of other treatments were all entered together in the multivariate model, clustered by general practice. We checked the proportional hazards assumption for included variables, and the only variable violating the assumption-dementia-was included as a stratifying factor. Continuous variables were assessed for non-linear risk association and were included into the model as fractional polynomial terms. 
To account for missing values, we used multiple imputation to create five imputed datasets with multiple chained equations, applying Rubin's rules to combine effect estimates and standard errors. ${ }^{24}$ The imputation model included all potentially important covariates, statin stopper status, years of records, and cumulative exposure to statins. ${ }^{25}$ To test our assumption that data were missing at random, we ran a sensitivity analysis using only records with complete data.

The second analysis of discontinuers to identify restarters used the same analytical approach as that used to identify the discontinuers. The model included the same covariates apart from statin type and dose use at baseline, which were replaced by statin type and dose at the time of discontinuation. The multivariate analysis of discontinuers to investigate restarting was not described in the protocol and was added for consistency.

\section{Additional analyses}

We conducted all six additional analyses using the same design with the exception of one with a changed definition of outcome. The first two analyses addressed possible differences between patients who discontinued within the first year and those who stayed on statin treatment for more than a year. They comprised one analysis of factors associated with statin discontinuation restricted to the first year of follow-up with patients censored at this point, and one analysis restricted to patients still taking statins at one year and analysing factors associated with statin discontinuation during the remaining follow-up time. low-up of the cohort at five years to reduce the effect of possible changes in baseline variables. In the fourth

Patients with at least 1 prescription in study period and 1 year of medical records before and after initiation of statin treatment $(n=887313)$

Patients with prescriptions in past 12 months before entering study ( $n=290511)$

Patients used in sensitivity analysis $(n=596802)$ :

Without cardiovascular disease $(n=454641)$

With cardiovascular disease $(n=142161)$

Patients with only 1 prescription excluded from main analysis $(n=26465)$ :

Without cardiovascular disease $(n=23618)$

With cardiovascular disease $(n=2847)$

Patients used in main analysis for

discontinuation $(n=570337)$

Without cardiovascular disease $(n=431023) \longrightarrow$ With cardiovascular disease $(n=139314)$

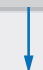

Patients discontinued, used in main analysis for restarting ( $n=262$ 413):

Without cardiovascular disease $(n=204622)$

With cardiovascular disease $(n=57791)$

$\downarrow$

Patients restarted ( $n=190$ 516):

Without cardiovascular disease $(n=147305)$

With cardiovascular disease $(n=43211)$

Fig 1 | Flow chart of study patients discontinuing and restarting statin treatment, based on CPRD data
Patients with at least 2 years of follow-up, used in sensitivity analysis for discontinued after 12 months gap $(n=508269)$ : Without cardiovascular disease $(n=384025)$ With cardiovascular disease $(n=124244)$ $\downarrow$

Patients discontinued, used in sensitivity analysis for restarting $(\mathrm{n}=136777)$ : Without cardiovascular disease $(n=109706)$ With cardiovascular disease $(n=27071)$

Patients restarted ( $\mathrm{n}=62$ 889):

Without cardiovascular disease $(n=49570)$ With cardiovascular disease $(n=13319)$
In the third additional analysis, we truncated the fol-

analysis, we included all patients-even those with only one prescription. We conducted the fifth analysis to facilitate comparison with studies defining discontinuation as a period substantially longer than 90 days. ${ }^{1926}$ In this analysis, we selected patients with at least two years of follow-up, defining discontinuation as the first 12 month gap after the estimated end date of any prescription, and included an analysis of restarting for discontinuers. In the sixth analysis, we did not assume that people without recorded ethnicity were white, adding a category for not recorded.

\section{Patient involvement}

In this study, patients were not involved in setting the research question or outcome measures, or in the design or implementation of the study. However, the patient reviewer for The BMJ provided helpful comments on interpretation of results and limitations of the study, and the paper was revised accordingly. With respect to dissemination, the patient reviewer noted that the study findings could highlight for patients the complexity of the issue and encourage them to discuss adherence problems with their doctor to try to discover solutions. Lay summaries associated with publication publicity will be created and any consequent media opportunities to disseminate these findings more widely will be taken up.

\section{Results}

We identified 570337 patients with at least two statin prescriptions during the study period and no statin prescription in the 12 months previous to the study, 431023 (76\%) in the primary prevention group and 139314 (24\%) in the secondary. Overall, in the primary prevention group, with a median follow-up time of 137 weeks, 204622 (47\%) discontinued statin treatment and 147305 ( $72 \%$ of those who discontinued) restarted. In the secondary prevention group, with a median follow-up time of 182 weeks, 57791 (41\%) discontinued and 43211 (75\% of those who discontinued) restarted (fig 1).

Within the primary prevention group, 12445 (3\%) patients were censored during follow-up because of a cardiovascular event. Of these, 566 (4.5\%) died within 90 days of diagnosis, 433 (3.5\%) within 28 days. Of patients with a longer survival time, 7322 (62\%) had a statin prescription within 28 days of their diagnosis, 11359 (96\%) within two months.

\section{Cohort description}

In this article, we present results for the primary and secondary prevention groups together to highlight similarities and differences. When both prevention groups are involved, the first number is for the primary group and the second for the secondary group. During follow-up, the overall discontinuation rates were 13 and 9.6 per 100 person years for the primary and secondary prevention groups, respectively. After the first six months, 19\% and 13\% had discontinued, after one year $27 \%$ and $19 \%$, and after two years $35 \%$ and $26 \%$ (fig 2). For patients discontinuing, restarting rates were 44 and 55 per 100 person years and median 


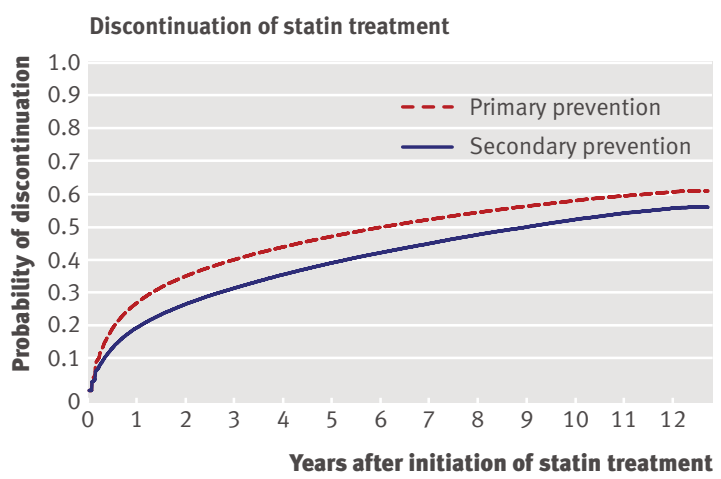

Fig 2 | Patients who discontinued statin treatment, based on CPRD data, October 2014

gaps between discontinuation and restarting were 36 weeks (interquartile range 18-108) and 29 weeks (17-77)). Within the discontinuation group, 38\% and $43 \%$ restarted within six months of stopping, 55\% and $61 \%$ within one year, and $66 \%$ and $72 \%$ within two years (fig 3).

\section{Statin prescribing}

Tables 1 and 2 present descriptive statistics for the primary and secondary groups, respectively. Most patients started treatment using simvastatin $(79 \%$ and $68 \%$ for the primary and secondary prevention groups, respectively), with fewer starting on atorvastatin (16\% and $25 \%$, respectively). Most users did not switch statins ( $81 \%$ and $78 \%$ ), but of those who did, $52 \%$ and $49 \%$ changed to atorvastatin, and $28 \%$ and $33 \%$ to simvastatin. At the time of stopping or leaving the cohort, $72 \%$ and $63 \%$ were still on simvastatin, and $21 \%$ and $28 \%$ on atorvastatin. Most restarters resumed on their most recently prescribed statin ( $78 \%$ and $80 \%)$, but of the $22 \%$ and $20 \%$ who changed statins, $42 \%$ and $37 \%$ switched to atorvastatin, and $29 \%$ and $37 \%$ to simvastatin . Among patients who restarted, the most common drug was simvastatin ( $67 \%$ and $63 \%$ ), followed by atorvastatin (22\% and $26 \%$; supplementary tables 1 and 2$)$.

In the analysis for discontinuation of statin treatment, type and dose of statin were not important factors in the primary prevention group (table 3). In the secondary prevention group, rosuvastatin use was

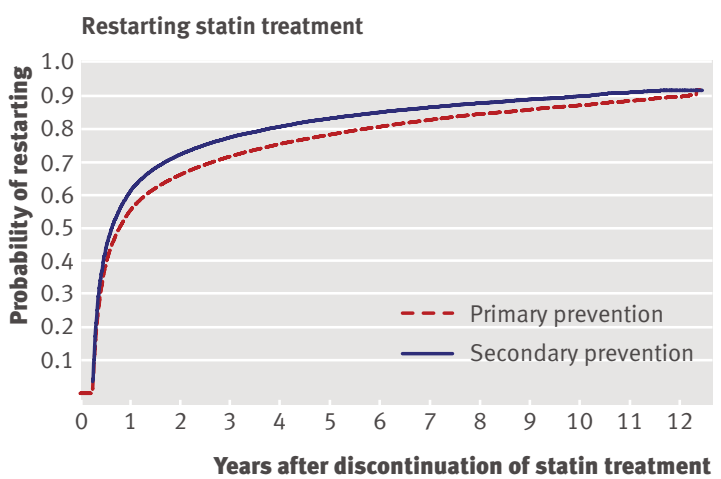

Fig 3 Patients who restarted statins after discontinuation, based on CPRD data, October 2014 associated with increased discontinuation risk, and pravastatin use was associated with decreased risk (both compared with simvastatin use; table 4). Patients on a lower statin dose were more likely to discontinue treatment, and patients on a higher dose less likely to discontinue. For patients restarting statin treatment in both the primary and secondary prevention groups, type and dose of statin in the final prescription before discontinuation were important factors (tables 3 and 4). Statins other than simvastatin were associated with a decreased risk of restarting. Patients on a lower statin dose were less likely to restart treatment, and patients on a higher dose more likely to restart.

\section{Factors associated with discontinuation of statin treatment}

Overall, patients in the primary prevention group were more likely to discontinue with statin treatment than those in the secondary prevention group (unadjusted hazard ratio 1.28 (95\% confidence interval 1.27 to 1.30), adjusted for sex and age 1.26 (1.24 to 1.27)).

\section{Increased risks of discontinuation}

Tables 3 and 4 show the hazard ratios for associations between the factors and discontinuation risk in primary and secondary prevention groups, and fig 4 presents the hazard ratios for continuous variables in both groups. In the multivariate model accounting for comorbidities, clinical values, and lifestyle variables, factors associated with increased risk of discontinuation in both the primary and secondary prevention groups were:

- Age younger than 50 years (age 50 years $v 60$ years; adjusted hazard ratio 1.16 (95\% confidence interval 1.15 to 1.16 ) for primary prevention group, 1.11 (1.10 to 1.13) for secondary prevention group)

- Age 75 years or older (age 75 years $v 60$ years; 1.04 (1.03 to 1.05) for primary prevention group, 1.10 (1.08 to 1.12) for secondary prevention group)

- Female sex

- Ethnic minority group (compared with white or non-recorded patients)

- Relatively low body mass index (index of $20 v$ index of 25; 1.09 (1.08 to 1.11) for primary prevention group, 1.12 (1.10 to 1.14) for secondary prevention group)

- Current smoking (compared with non-smokers)

- Type 1 diabetes

- Chronic liver disease

- Chronic obstructive pulmonary disease

- Baseline total cholesterol/high density lipoprotein ratio above 5 (ratio $5.25 v$ ratio 3.5; 1.11 (1.10 to 1.12) for primary prevention group, 1.10 (1.08 to 1.12$)$ for secondary prevention group)

- Prescriptions for other treatments

- In patients who had used statins for more than 12 months, dementia was associated with an increased risk of subsequent discontinuation.

Factors associated with increased risk of discontinuation in the secondary prevention group only 
Table 1 | Discontinuation of statin treatment in primary prevention group. Descriptive statistics shown for all included patients, patients who continued, patients who discontinued, and patients who restarted statin treatment. Data are No (\%) of patients unless stated otherwise. SD=standard deviation Initiated $(\mathrm{n}=431023)$

Continued $(n=226401)$

Discontinued $(n=204622)$

Restarted ( $n=147305)$

Age at study entry

\begin{tabular}{|c|c|c|c|c|}
\hline$<45$ years & $31290(7.3)$ & $12303(5.4)$ & $18987(9.3)$ & $14627(9.9)$ \\
\hline 45-54 years & $77770(18.0)$ & $37191(16.4)$ & 40579 (19.8) & $31203(21.2)$ \\
\hline $55-64$ years & $140042(32.5)$ & $76395(33.7)$ & $63647(31.1)$ & $47593(32.3)$ \\
\hline $65-74$ years & $129429(30.0)$ & 72574 (32.1) & $56855(27.8)$ & $39366(26.7)$ \\
\hline $75-84$ years & $52492(12.2)$ & $27938(12.3)$ & $24554(12.0)$ & $14516(9.9)$ \\
\hline Mean (SD; years) & $61.7(11.0)$ & $62.5(10.4)$ & $60.8(11.6)$ & $59.9(11.4)$ \\
\hline \multicolumn{5}{|l|}{ Sex } \\
\hline Men & $225732(52.4)$ & $120423(53.2)$ & $105309(51.5)$ & 77786 (52.8) \\
\hline Women & $205291(47.6)$ & $105978(46.8)$ & $99313(48.5)$ & $69519(47.2)$ \\
\hline \multicolumn{5}{|l|}{ Ethnicity } \\
\hline Ethnicity recorded & $277935(64.5)$ & $144238(63.7)$ & $133697(65.3)$ & $96516(65.5)$ \\
\hline White or not recorded & $415392(96.4)$ & 220234 (97.3) & $195158(95.4)$ & $139549(94.7)$ \\
\hline Indian & $4306(1.0)$ & $1730(0.8)$ & $2576(1.3)$ & $2160(1.5)$ \\
\hline Pakistani & $1786(0.4)$ & $672(0.3)$ & $1114(0.5)$ & $944(0.6)$ \\
\hline Bangladeshi & $642(0.1)$ & $265(0.1)$ & $377(0.2)$ & $319(0.2)$ \\
\hline Other Asian & $1881(0.4)$ & $783(0.3)$ & $1098(0.5)$ & $884(0.6)$ \\
\hline Black African & $1358(0.3)$ & $457(0.2)$ & $901(0.4)$ & $721(0.5)$ \\
\hline Black Caribbean & $1882(0.4)$ & $633(0.3)$ & $1249(0.6)$ & $1044(0.7)$ \\
\hline Chinese & $619(0.1)$ & $283(0.1)$ & $336(0.2)$ & $257(0.2)$ \\
\hline Other ethnic group & $3157(0.7)$ & $1344(0.6)$ & $1813(0.9)$ & $1427(1.0)$ \\
\hline \multicolumn{5}{|l|}{ Smoking status } \\
\hline Patients with smoking status recorded & $430792(99.9)$ & $226287(99.9)$ & 204505 (99.9) & $147254(100)$ \\
\hline Non-smoker & $213066(49.4)$ & 112101 (49.5) & 100965 (49.3) & $72160(49.0)$ \\
\hline Ex-smoker & $132685(30.8)$ & $73179(32.3)$ & $59506(29.1)$ & $42114(28.6)$ \\
\hline Light smoker & $17462(4.1)$ & $8636(3.8)$ & $8826(4.3)$ & $6569(4.5)$ \\
\hline Moderate smoker & $45214(10.5)$ & $22087(9.8)$ & $23127(11.3)$ & $17189(11.7)$ \\
\hline Heavy smoker & $22365(5.2)$ & $10284(4.5)$ & $12081(5.9)$ & $9222(6.3)$ \\
\hline \multicolumn{5}{|l|}{ Clinical measurements } \\
\hline Patients with body mass index recorded & $419303(97.3)$ & $220599(97.4)$ & $198704(97.1)$ & $143475(97.4)$ \\
\hline Body mass index (mean (SD)) & $28.9(5.5)$ & $29.0(5.5)$ & $28.8(5.5)$ & $29.0(5.5)$ \\
\hline Patients with systolic blood pressure recorded & $430891(100)$ & $226358(100)$ & $204533(100)$ & $147264(100)$ \\
\hline Systolic blood pressure (mean (SD)) & $141.0(18.3)$ & $141.5(18.2)$ & $140.5(18.5)$ & $140.5(18.4)$ \\
\hline Patients with ratio recorded & $283855(65.9)$ & $152720(67.5)$ & $131135(64.1)$ & $92820(63.0)$ \\
\hline Total cholesterol:high density lipoprotein ratio (mean (SD)) & $4.4(1.5)$ & $4.3(1.5)$ & $4.5(1.5)$ & $4.5(1.5)$ \\
\hline \multicolumn{5}{|c|}{ Chronic conditions } \\
\hline Rheumatoid arthritis & $6684(1.6)$ & $3459(1.5)$ & $3225(1.6)$ & $2256(1.5)$ \\
\hline Chronic renal disease & $2916(0.7)$ & $1413(0.6)$ & $1503(0.7)$ & $1039(0.7)$ \\
\hline Liver disease & $2017(0.5)$ & $988(0.4)$ & $1029(0.5)$ & $708(0.5)$ \\
\hline Chronic obstructive pulmonary disease & $15294(3.5)$ & $8266(3.7)$ & $7028(3.4)$ & $4766(3.2)$ \\
\hline Cancer & $32365(7.5)$ & $17551(7.8)$ & $14814(7.2)$ & $9670(6.6)$ \\
\hline Atrial fibrillation & $13754(3.2)$ & $8043(3.6)$ & $5711(2.8)$ & $3641(2.5)$ \\
\hline Hypertension & $217632(50.5)$ & $122573(54.1)$ & $95059(46.5)$ & $68418(46.4)$ \\
\hline Heart failure & $5078(1.2)$ & $2954(1.3)$ & $2124(1.0)$ & $1338(0.9)$ \\
\hline Type 1 diabetes & $9960(2.3)$ & $4420(2.0)$ & $5540(2.7)$ & $4632(3.1)$ \\
\hline Type 2 diabetes & $100848(23.4)$ & $56338(24.9)$ & $44510(21.8)$ & $35359(24.0)$ \\
\hline Dementia & $1629(0.4)$ & $990(0.4)$ & $639(0.3)$ & $282(0.2)$ \\
\hline \multicolumn{5}{|l|}{ Genetic characteristics } \\
\hline Family history of premature coronary heart disease & $30734(7.1)$ & $16158(7.1)$ & $14576(7.1)$ & $10731(7.3)$ \\
\hline Familial hypercholesterolaemia & $1364(0.3)$ & $699(0.3)$ & $665(0.3)$ & $504(0.3)$ \\
\hline \multicolumn{5}{|l|}{ Use of other treatments } \\
\hline Aspirin & $102743(23.8)$ & $54467(24.1)$ & $48276(23.6)$ & $33316(22.6)$ \\
\hline Anticoagulants & $11906(2.8)$ & $7030(3.1)$ & $4876(2.4)$ & $3150(2.1)$ \\
\hline No of other non-cardiovascular treatments & $2.0(1.6)$ & $2.0(1.6)$ & $2.1(1.6)$ & $2.0(1.6)$ \\
\hline \multicolumn{5}{|l|}{ QRISK2 score } \\
\hline $0-4 \%$ & $30062(7.0)$ & $11473(5.1)$ & $18589(9.1)$ & $13368(9.1)$ \\
\hline $5-9 \%$ & $54066(12.5)$ & $25802(11.4)$ & $28264(13.8)$ & $21032(14.3)$ \\
\hline $10-14 \%$ & $59495(13.8)$ & $30897(13.6)$ & $28598(14.0)$ & $21279(14.4)$ \\
\hline $15-19 \%$ & $58358(13.5)$ & 31541 (13.9) & $26817(13.1)$ & $19877(13.5)$ \\
\hline $20-24 \%$ & $52020(12.1)$ & 28629 (12.6) & $23391(11.4)$ & $17124(11.6)$ \\
\hline $25-99 \%$ & $177022(41.1)$ & 98059 (43.3) & $78963(38.6)$ & $54625(37.1)$ \\
\hline Mean (SD; \%) & $24.5(16.2)$ & $25.4(16.0)$ & $23.5(16.4)$ & $22.9(16.0)$ \\
\hline
\end{tabular}




\begin{tabular}{|c|c|c|c|c|}
\hline & Initiated $(n=431023)$ & Continued $(n=226401)$ & Discontinued $(n=204622)$ & Restarted $(n=147305)$ \\
\hline \multicolumn{5}{|l|}{ Townsend score (patient based) } \\
\hline Patients with data recorded & $266055(61.7)$ & $139487(61.6)$ & $126568(61.9)$ & $91036(61.8)$ \\
\hline Group 1 (most affluent) & $65226(15.1)$ & $34761(15.4)$ & $30465(14.9)$ & $21580(14.6)$ \\
\hline Group 2 & $62581(14.5)$ & $33141(14.6)$ & $29440(14.4)$ & $20684(14.0)$ \\
\hline Group 3 & $54715(12.7)$ & $28522(12.6)$ & $26193(12.8)$ & $18666(12.7)$ \\
\hline Group 4 & $50631(11.7)$ & $26496(11.7)$ & $24135(11.8)$ & $17653(12.0)$ \\
\hline Group 5 (most deprived) & $32902(7.6)$ & $16567(7.3)$ & $16335(8.0)$ & $12453(8.5)$ \\
\hline \multicolumn{5}{|l|}{ Townsend score (practice based) } \\
\hline Group 1 (most affluent) & $77164(17.9)$ & $40086(17.7)$ & 37078 (18.1) & $26508(18.0)$ \\
\hline Group 2 & $81744(19.0)$ & $42737(18.9)$ & $39007(19.1)$ & $28114(19.1)$ \\
\hline Group 3 & $87529(20.3)$ & $46375(20.5)$ & $41154(20.1)$ & $29242(19.9)$ \\
\hline Group 4 & $94933(22.0)$ & $49729(22.0)$ & $45204(22.1)$ & $32570(22.1)$ \\
\hline Group 5 (most deprived) & $89653(20.8)$ & $47474(21.0)$ & $42179(20.6)$ & $30871(21.0)$ \\
\hline \multicolumn{5}{|l|}{ Statin use at baseline } \\
\hline Simvastatin & $342384(79.4)$ & $181249(80.1)$ & $161135(78.7)$ & $114281(77.6)$ \\
\hline Atorvastatin & $69854(16.2)$ & $35655(15.7)$ & $34199(16.7)$ & $25975(17.6)$ \\
\hline Pravastatin & $9408(2.2)$ & $4767(2.1)$ & $4641(2.3)$ & $3505(2.4)$ \\
\hline Rosuvastatin & $7937(1.8)$ & $4014(1.8)$ & $3923(1.9)$ & $2985(2.0)$ \\
\hline Fluvastatin & $1440(0.3)$ & $716(0.3)$ & $724(0.4)$ & $559(0.4)$ \\
\hline \multicolumn{5}{|c|}{ Statin dose at baseline (potency units) } \\
\hline Less than 1 & $51830(12.0)$ & $25929(11.5)$ & $25901(12.7)$ & $18969(12.9)$ \\
\hline One & $183541(42.6)$ & 94815 (41.9) & $88726(43.4)$ & $65015(44.1)$ \\
\hline Two & $190623(44.2)$ & $103025(45.5)$ & $87598(42.8)$ & $61613(41.8)$ \\
\hline Three and more & $5029(1.2)$ & $2632(1.2)$ & $2397(1.2)$ & $1708(1.2)$ \\
\hline
\end{tabular}

were: atrial fibrillation; familial hypercholesterolaemia (tables 3 and 4, fig 4).

\section{Decreased risk of discontinuation}

In both prevention groups, factors associated with decreased risk of discontinuation of statin treatment were ex-smokers, hypertension, and use of anticoagulant drugs. For the primary prevention group only, these factors were type 2 diabetes, atrial fibrillation, heart failure, and familial hypercholesterolaemia. For the secondary prevention group only, aspirin use was associated with decreased risk. In both groups, dementia was associated with a decreased risk for the first 12 months of follow-up.

\section{Factors associated with restarting of statin} treatment

Overall, patients who had stopped taking statins in the primary prevention group were less likely to restart statin treatment than those in the secondary prevention group (unadjusted hazard ratio 0.86 ( $95 \%$ confidence interval 0.84 to 0.87 ); adjusted hazard ratio for sex and age 0.80 (0.79 to 0.81$)$ ).

\section{Increased risk of restarting}

In the multivariate model accounting for comorbidities, clinical values, and lifestyle variables, factors associated with an increased risk of restarting (that is, being more likely to restart) in both the primary and secondary prevention groups were ethnic minority groups (compared with white or nonrecorded patients), current smoking, and type 2 diabetes. Factors associated with increased risk of restarting in the primary prevention group only were type 1 diabetes, chronic obstructive pulmonary disease, hypertension, family history of cardiovascular disease, and familial hypercholesterolaemia. In the secondary prevention group only, these factors were younger age, ex-smoking, and aspirin use (tables 3 and 4 , fig 5).

\section{Decreased risk of restarting}

Tables 3 and 4 show the hazard ratios for associations between the factors and risk of restarting in primary and secondary prevention groups, and fig 5 presents the hazard ratios for continuous variables in both groups. Factors associated with a decreased risk of restarting (less likely to restart) in both the primary and secondary prevention groups were female sex, age 75 years or older (age 75 years $v 60$ years, adjusted hazard ratio 0.90 (95\% confidence interval 0.89 to 0.91 ) for primary prevention group, 0.86 ( 0.85 to 0.87$)$ for secondary prevention group), and dementia. Factors associated with a decreased risk of restarting in the primary prevention group only were:

- Age 40 years or younger (age 40 years $v 60$ years, 0.96 (0.95 to 0.97))

- Cancer

- Aspirin use

- Anticoagulant use

- Relatively low body mass index (index $20 v$ index 25, 0.96 (0.95 to 0.97))

- Baseline total cholesterol:high density lipoprotein ratio below 1.4 (ratio $1.4 v$ ratio 3.5, 0.90 (0.88 to 0.92$)$ ). 
Table 2 | Discontinuation of statin treatment in secondary prevention group. Descriptive statistics shown for all included patients, patients who continued, who discontinued, and patients who restarted statin treatment. Data are no (\%) of patients unless stated otherwise. SD=standard deviation Initiated $(\mathrm{n}=139314)$ Continued $(n=81523)$ Discontinued $(\mathrm{n}=57791) \quad$ Restarted $(\mathrm{n}=43211)$

Age at study entry

\begin{tabular}{|c|c|c|c|c|}
\hline$<45$ years & $4158(3.0)$ & $2167(2.7)$ & $1991(3.4)$ & $1629(3.8)$ \\
\hline 45-54 years & $15051(10.8)$ & $8923(10.9)$ & $6128(10.6)$ & $5063(11.7)$ \\
\hline 55-64 years & $32620(23.4)$ & $19995(24.5)$ & $12625(21.8)$ & $10236(23.7)$ \\
\hline $65-74$ years & $44055(31.6)$ & $26113(32.0)$ & $17942(31.0)$ & $13580(31.4)$ \\
\hline $75-84$ years & $43430(31.2)$ & $24325(29.8)$ & $19105(33.1)$ & $12703(29.4)$ \\
\hline Mean (SD; years) & $67.4(11.0)$ & $67.2(10.8)$ & $67.7(11.2)$ & $66.8(11.3)$ \\
\hline \multicolumn{5}{|l|}{ Sex } \\
\hline Men & $82485(59.2)$ & $50353(61.8)$ & $32132(55.6)$ & $24472(56.6)$ \\
\hline Women & $56829(40.8)$ & $31170(38.2)$ & $25659(44.4)$ & $18739(43.4)$ \\
\hline \multicolumn{5}{|l|}{ Ethnicity } \\
\hline Ethnicity recorded & $93587(67.2)$ & $54495(66.8)$ & $39092(67.6)$ & $29332(67.9)$ \\
\hline White or not recorded & $136259(97.8)$ & $79986(98.1)$ & $56273(97.4)$ & 41961 (97.1) \\
\hline Indian & $953(0.7)$ & $479(0.6)$ & $474(0.8)$ & $394(0.9)$ \\
\hline Pakistani & $422(0.3)$ & $218(0.3)$ & $204(0.4)$ & $174(0.4)$ \\
\hline Bangladeshi & $121(0.1)$ & $67(0.1)$ & $54(0.1)$ & $50(0.1)$ \\
\hline Other Asian & $229(0.2)$ & $106(0.1)$ & $123(0.2)$ & $102(0.2)$ \\
\hline Black African & $165(0.1)$ & $79(0.1)$ & $86(0.1)$ & $69(0.2)$ \\
\hline Black Caribbean & $368(0.3)$ & $146(0.2)$ & $222(0.4)$ & $187(0.4)$ \\
\hline Chinese & $123(0.1)$ & $71(0.1)$ & $52(0.1)$ & $41(0.1)$ \\
\hline Other ethnic group & $674(0.5)$ & $371(0.5)$ & $303(0.5)$ & $233(0.5)$ \\
\hline \multicolumn{5}{|l|}{ Smoking status } \\
\hline Patients with smoking status recorded & $139233(99.9)$ & $81479(99.9)$ & $57754(99.9)$ & $43194(100.0)$ \\
\hline Non-smoker & $59686(42.8)$ & $34565(42.4)$ & $25121(43.5)$ & $18350(42.5)$ \\
\hline Ex-smoker & $56325(40.4)$ & $34288(42.1)$ & $22037(38.1)$ & $16445(38.1)$ \\
\hline Light smoker & $5690(4.1)$ & $3157(3.9)$ & $2533(4.4)$ & $2020(4.7)$ \\
\hline Moderate smoker & $12281(8.8)$ & $6752(8.3)$ & $5529(9.6)$ & $4324(10.0)$ \\
\hline Heavy smoker & $5251(3.8)$ & $2717(3.3)$ & $2534(4.4)$ & $2055(4.8)$ \\
\hline \multicolumn{5}{|l|}{ Clinical measurements } \\
\hline Patients with body mass index recorded & $134436(96.5)$ & $78635(96.5)$ & $55801(96.6)$ & $41982(97.2)$ \\
\hline Body mass index (mean (SD)) & $27.6(5.1)$ & $27.7(5.0)$ & $27.5(5.2)$ & $27.7(5.2)$ \\
\hline Patients with systolic blood pressure recorded & $139283(100)$ & $81508(100.0)$ & $57775(100.0)$ & $43209(100)$ \\
\hline Systolic blood pressure (mean (SD)) & $137.8(20.3)$ & $137.1(20.4)$ & $138.7(20.2)$ & $138.7(20.2)$ \\
\hline Patients with ratio recorded & $70278(50.4)$ & $42124(51.7)$ & $28154(48.7)$ & $20679(47.9)$ \\
\hline Total cholesterol:high density lipoprotein ratio (mean (SD)) & $3.8(1.3)$ & $3.8(1.3)$ & $3.9(1.3)$ & $3.9(1.3)$ \\
\hline \multicolumn{5}{|l|}{ Chronic conditions } \\
\hline Rheumatoid arthritis & $2950(2.1)$ & $1663(2.0)$ & $1287(2.2)$ & $930(2.2)$ \\
\hline Chronic renal disease & $1131(0.8)$ & $623(0.8)$ & $508(0.9)$ & $331(0.8)$ \\
\hline Liver disease & $757(0.5)$ & $405(0.5)$ & $352(0.6)$ & $245(0.6)$ \\
\hline Chronic obstructive pulmonary disease & $9058(6.5)$ & $4892(6.0)$ & $4166(7.2)$ & $3030(7.0)$ \\
\hline Cancer & $14090(10.1)$ & 8092 (9.9) & $5998(10.4)$ & $4077(9.4)$ \\
\hline Atrial fibrillation & $12091(8.7)$ & $7030(8.6)$ & $5061(8.8)$ & $3388(7.8)$ \\
\hline Hypertension & $63610(45.7)$ & $36824(45.2)$ & $26786(46.3)$ & $19723(45.6)$ \\
\hline Heart failure & $8693(6.2)$ & $5169(6.3)$ & $3524(6.1)$ & $2397(5.5)$ \\
\hline Type 1 diabetes & $1501(1.1)$ & $805(1.0)$ & $696(1.2)$ & $535(1.2)$ \\
\hline Type 2 diabetes & $15472(11.1)$ & $8858(10.9)$ & $6614(11.4)$ & $4949(11.5)$ \\
\hline Dementia & $1165(0.8)$ & $729(0.9)$ & $436(0.8)$ & $220(0.5)$ \\
\hline \multicolumn{5}{|l|}{ Genetic characteristics } \\
\hline Family history of premature coronary heart disease & $8076(5.8)$ & $4800(5.9)$ & $3276(5.7)$ & $2537(5.9)$ \\
\hline Familial hypercholesterolaemia & $46(0.0)$ & $19(0.0)$ & $27(0.0)$ & $20(0.0)$ \\
\hline \multicolumn{5}{|l|}{ Use of other treatments } \\
\hline Aspirin & $101146(72.6)$ & $60684(74.4)$ & $40462(70.0)$ & $30179(69.8)$ \\
\hline Anticoagulants & $10744(7.7)$ & $6354(7.8)$ & $4390(7.6)$ & $3023(7.0)$ \\
\hline No of other non-cardiovascular treatments & $2.3(1.6)$ & $2.3(1.6)$ & $2.4(1.6)$ & $2.4(1.6)$ \\
\hline \multicolumn{5}{|l|}{ Townsend score (patient based) } \\
\hline Patients with data recorded & $84404(60.6)$ & $49293(60.5)$ & $35111(60.8)$ & $26092(60.4)$ \\
\hline Group 1 (most affluent) & $18966(13.6)$ & $11286(13.8)$ & $7680(13.3)$ & $5613(13.0)$ \\
\hline Group 2 & $19647(14.1)$ & $11491(14.1)$ & $8156(14.1)$ & $5922(13.7)$ \\
\hline Group 3 & $17905(12.9)$ & $10544(12.9)$ & $7361(12.7)$ & 5439 (12.6) \\
\hline Group 4 & $16898(12.1)$ & $9639(11.8)$ & $7259(12.6)$ & $5503(12.7)$ \\
\hline Group 5 (most deprived) & $10988(7.9)$ & $6333(7.8)$ & $4655(8.1)$ & $3615(8.4)$ \\
\hline
\end{tabular}




\begin{tabular}{|c|c|c|c|c|}
\hline & Initiated $(n=139314)$ & Continued $(n=81523)$ & Discontinued $(n=57791)$ & Restarted $(n=43211)$ \\
\hline \multicolumn{5}{|c|}{ Townsend score (practice based) } \\
\hline Group 1 (most affluent) & $22887(16.4)$ & $13399(16.4)$ & $9488(16.4)$ & $7057(16.3)$ \\
\hline Group 2 & $25379(18.2)$ & $14988(18.4)$ & $10391(18.0)$ & $7811(18.1)$ \\
\hline Group 3 & $28498(20.5)$ & $16669(20.4)$ & $11829(20.5)$ & $8673(20.1)$ \\
\hline Group 4 & $32131(23.1)$ & $18752(23.0)$ & $13379(23.2)$ & $10002(23.1)$ \\
\hline Group 5 (most deprived) & $30419(21.8)$ & $17715(21.7)$ & $12704(22.0)$ & $9668(22.4)$ \\
\hline \multicolumn{5}{|l|}{ Statin use at baseline } \\
\hline Simvastatin & $95198(68.3)$ & $55598(68.2)$ & $39600(68.5)$ & $29194(67.6)$ \\
\hline Atorvastatin & $34155(24.5)$ & $20653(25.3)$ & $13502(23.4)$ & $10405(24.1)$ \\
\hline Pravastatin & $6858(4.9)$ & $3656(4.5)$ & $3202(5.5)$ & $2446(5.7)$ \\
\hline Rosuvastatin & $2236(1.6)$ & $1199(1.5)$ & $1037(1.8)$ & $802(1.9)$ \\
\hline Fluvastatin & $867(0.6)$ & $417(0.5)$ & $450(0.8)$ & $364(0.8)$ \\
\hline \multicolumn{5}{|c|}{ Statin dose at baseline (potency units) } \\
\hline Less than 1 & $16397(11.8)$ & $8202(10.1)$ & $8195(14.2)$ & $6210(14.4)$ \\
\hline One & $52159(37.4)$ & $28090(34.5)$ & $24069(41.6)$ & $18315(42.4)$ \\
\hline Two & $60512(43.4)$ & $37509(46.0)$ & $23003(39.8)$ & $16826(38.9)$ \\
\hline Three and more & $10246(7.4)$ & $7722(9.5)$ & $2524(4.4)$ & $1860(4.3)$ \\
\hline
\end{tabular}

For the secondary prevention group only, the additional factor was atrial fibrillation.

\section{Categorised results summary}

All of the associations between patient factors and increased, decreased, or similar risks of discontinuation and restarting statin treatment were categorised into three groups (table 5):

- Patients who are more likely to discontinue and less likely to restart-that is, elevated discontinuation and lowered or neutral restarting, or neutral discontinuation and lowered restarting (real stopping)

- Patients who are both more likely to discontinue and more likely to restart-that is, elevated discontinuation and elevated restarting (stopping-restarting)

- Patients who are less likely to discontinue or more likely to restart-that is, lowered discontinuation and elevated or neutral restarting, or neutral discontinuation and elevated restarting (relative adherence).

\section{Additional analyses \\ Terms of use}

Statin discontinuation rates were much higher in the first year of use (32 and 21 per 100 person years) than after the first year of use (7.4 and 6.4 per 100 person years). Results from the separate analyses for factors associated with discontinuation in the first year of use and after the first year were all consistent in both primary and secondary prevention groups for all factors, apart from the finding for dementia described earlier (supplementary table 3).

Second definition of discontinuation (12 month gap) We identified 384025 patients with at least two years of follow-up in the primary prevention group and 124244 patients in the secondary prevention group. Overall, in the primary prevention group, with a median follow-up time of 235 weeks, 109706 (29\%) discontinued with a rate of 5.9 per 100 person years and 49570 (45\% of those who discontinued) restarted. In the secondary prevention group, with a medium of 282 weeks, 27071 (22\%) discontinued with a rate of 3.8 per 100 patient years and 13319 (49\% of those who discontinued) restarted the treatment by the end of the study period (supplementary figs 1 and 2).

Factors associated with increased or decreased risks of discontinuation and restarting were broadly consistent with the main analysis. Only for black ethnicity and only in the primary prevention group was the risk of discontinuation statistically significant, but the risk of restarting did increase for all ethnic minorities in the primary prevention group and for some in the secondary prevention group. These results accord with those from the main analysis (supplementary table 5, supplementary figs 3 and 4).

\section{Sensitivity analyses}

In both prevention groups, inclusion of patients with only one statin prescription resulted in higher overall rates of discontinuation, with 228240 (50\%) and 60638 (43\%) of patients discontinuing during the study period. The analysis of factors associated with discontinuation, however, gave similar results to the main analysis (supplementary table 6). Similar results were also seen in the analysis with an observational period ending not later than five years from the start of treatment, as well as the analysis with a separate category for unknown ethnicity (data not shown). Results from the analysis run on subgroups of patients with known Townsend score were in line with the main findings (supplementary table 4).

\section{Discussion}

In this large population based study, we used routinely collected data from primary care to determine discontinuation and restarting rates for statins, and to identify factors associated with higher or lower likelihoods of discontinuation or restarting of statin treatment. High rates of statin discontinuation have 


\section{Table 3 | Adjusted hazard ratios for discontinuation and restarting statin treatment in primary prevention group}

Adjusted hazard ratio $(95 \% \mathrm{Cl})$

Discontinuation*

Restarting $\dagger$

Sex

Men

Women

Ethnicity

White or not recorded

Indian

Pakistani

Bangladeshi

Other Asian

Black African

Black Caribbean

Chinese

Other ethnic group

Smoking status

Non-smoker

Ex-smoker

Light smoker

Moderate smoker

Heavy smoker

Chronic conditions

Rheumatoid arthritis

Chronic renal disease

Liver disease

Chronic obstructive pulmonary disease

Cancer

Atrial fibrillation

Hypertension

Heart failure

Type 1 diabetes

Type 2 diabetes

Dementia, short term statin use $\neq$

Dementia, long term statin usef

Dementia in previous statin use

Reference

1.06 (1.05 to 1.07$)$

Reference

0.92 (0.91 to 0.93)

Reference

1.45 (1.39 to 1.52$)$

1.48 (1.39 to 1.58$)$

1.18 (0.99 to 1.41$)$

1.37 (1.26 to 1.49$)$

1.89 (1.72 to 2.08)

1.89 (1.74 to 2.04$)$

1.25 (1.11 to 1.41 )

1.36 (1.28 to 1.44$)$

Reference

0.97 (0.96 to 0.98)

1.07 (1.04 to 1.09 )

1.10 (1.08 to 1.12 )

1.16 (1.13 to 1.19$)$

1.02 (0.99 to 1.06$)$

1.03 (0.98 to 1.08$)$

1.17 (1.10 to 1.25$)$

1.04 (1.01 to 1.06$)$

1.02 (1.00 to 1.04$)$

0.96 (0.93 to 0.99$)$

0.80 (0.79 to 0.81$)$

0.92 (0.88 to 0.97 )

1.18 (1.15 to 1.22$)$

0.80 (0.78 to 0.81$)$

0.63 (0.56 to 0.71 )

1.14 (1.03 to 1.26 )

Reference

1.30 (1.24 to 1.37)

1.29 (1.20 to 1.39$)$

1.46 (1.29 to 1.65$)$

1.26 (1.19 to 1.34$)$

1.30 (1.19 to 1.41$)$

1.30 (1.22 to 1.39$)$

1.26 (1.12 to 1.43$)$

1.21 (1.14 to 1.29$)$

Genetic characteristics

Family history of premature coronary heart disease

Familial hypercholesterolaemia

0.98 (0.96 to 1.00$)$

0.86 (0.80 to 0.93$)$

1.02 (1.01 to 1.04$)$

0.88 (0.85 to 0.91$)$

1.02 (1.02 to 1.03$)$

Reference

1.00 (0.98 to 1.01$)$

1.09 (1.06 to 1.12$)$

1.05 (1.04 to 1.07$)$

1.05 (1.03 to 1.08$)$

1.01 (0.96 to 1.06$)$

1.04 (0.97 to 1.12$)$

0.91 (0.85 to 0.98$)$

1.06 (1.03 to 1.10$)$

$0.97(0.95$ to 0.99$)$

$0.98(0.94$ to 1.02$)$

1.08 (1.07 to 1.10$)$

1.01 (0.96 to 1.07$)$

1.15 (1.11 to 1.19$)$

1.35 (1.33 to 1.37$)$

$-$

$0.80(0.71$ to 0.90$)$

1.04 (1.02 to 1.07$)$

$1.23(1.12$ to 1.35$)$

Aspirin

Anticoagulants

Each of other non-cardiovascular treatment

Reference

$\frac{\text { Townsend score group }}{1 \text { (most affluent) }}$

2

3

4

5 (most deprived)

1.02 (1.00 to 1.04$)$

$1.02(1.00$ to 1.05$)$

0.99 (0.96 to 1.02$)$

0.98 (0.95 to 1.02$)$

0.92 (0.91 to 0.94)

$0.94(0.90$ to 0.98$)$

1.00 (1.00 to 1.01$)$

Statin use at baseline

\begin{tabular}{ll}
\hline Simvastatin & Reference \\
\hline Atorvastatin & $0.99(0.97$ to 1.01$)$ \\
\hline Pravastatin & $0.98(0.94$ to 1.03$)$ \\
\hline Rosuvastatin & $0.96(0.91$ to 1.02$)$ \\
\hline Fluvastatin & 1.00 (0.92 to 1.09$)$ \\
\hline Statin dose at baseline (potency units) & \\
\hline Less than 1 & $1.02(1.00$ to 1.04$)$ \\
\hline One & Reference \\
\hline Two & 0.99 (0.98 to 1.01$)$ \\
\hline Three and more & $1.01(0.97$ to 1.06$)$
\end{tabular}

Reference

$0.99(0.97$ to 1.02$)$

$1.00(0.97$ to 1.03$)$

$1.02(0.99$ to 1.05$)$

1.08 (1.04 to 1.12 )

Reference

0.86 (0.85 to 0.88$)$

$0.84(0.81$ to 0.88$)$

0.81 ( 0.77 to 0.84$)$

0.85 (0.78 to 0.94$)$

0.89 (0.87 to 0.91$)$

Reference

1.11 (1.10 to 1.13$)$

1.41 (1.36 to 1.45$)$

All adjusted models include sex, ethnicity, smoking status, chronic conditions, use of other drugs, practice based Townsend score groups, and year of entering the study.

* Model also includes type and dose of statin at baseline, fractional polynomials for age ((age/5) and (age/5)2ln(age/5)), body mass index (BMI; BMI-0.5, In (BMI)), total cholesterol:high density

lipoprotein ratio (In(ratio)), and systolic blood pressure (SBP; (SBP/10), (SBP/10)2). Practice ID was included as clustering variable.

tModel also includes type and dose of statin at discontinuation, fractional polynomials for age $\left((\operatorname{age} / 5)^{0.5}\right.$, (age/5) $\left.{ }^{3}\right)$, body mass index (BMI-0.5), total cholesterol:high density lipoprotein: ratio

(ratio $\left.{ }^{0.5}\right)$, and systolic blood pressure ((SBP/10) $\left.)^{3}\right)$. Practice ID was included as clustering variable.

₹The analysis was stratified by dementia. Results for discontinuation were taken from additional analyses by terms of use (supplementary table 3).

$\S$ Estimates of Townsend score groups (patient based) are from the additional analysis (supplementary table 4). 


\section{Table 4 | Adjusted hazard ratios for discontinuation and restarting statin treatment in secondary prevention group \\ Adjusted hazard ratio $(95 \% \mathrm{Cl})$}

Discontinuation*

Restarting†

Sex

Men

Women

Ethnicity

White or not recorded

Indian

Pakistani

Bangladeshi

Other Asian

Black African

Black Caribbean

Chinese

Other ethnic group

Smoking status

Non-smoker

Ex-smoker

Light smoker

Moderate smoker

Heavy smoker

Chronic conditions

Rheumatoid arthritis

Chronic renal disease

Liver disease

Chronic obstructive pulmonary disease

Cancer

Atrial fibrillation

Hypertension

Heart failure

Type 1 diabetes

Type 2 diabetes

Dementia, short term statin use $\neq$

Dementia, long term statin use $\neq$

Dementia in previous statin use

Genetic characteristics

Family history of premature coronary heart disease

Familial hypercholesterolaemia

Use of other treatments

Aspirin

Anticoagulants

Each of other non-cardiovascular treatment

Townsend score groups (patient based)§

\begin{tabular}{|ll}
\hline 1 (most affluent) & Reference \\
\hline 2 & $1.02(0.98$ to 1.06$)$ \\
\hline 3 & $0.99(0.95$ to 1.03$)$ \\
\hline 4 & $1.02(0.97$ to 1.06$)$ \\
\hline 5 (most deprived) & $0.97(0.92$ to 1.01$)$ \\
\hline Statin use at baseline & Reference \\
\hline Simvastatin & $1.00(0.97$ to 1.03$)$ \\
\hline Atorvastatin & $0.94(0.90$ to 0.98$)$ \\
\hline Pravastatin & $1.19(1.11$ to 1.28$)$ \\
\hline Rosuvastatin & $1.09(0.99$ to 1.22$)$ \\
\hline Fluvastatin & \\
\hline Statin dose at baseline (potency units) & $1.11(1.08$ to 1.14$)$ \\
\hline Less than 1 & Reference \\
\hline One & $0.86(0.84$ to 0.88$)$ \\
\hline Two & 0.60 (0.57 to 0.64$)$ \\
\hline Three and more & .
\end{tabular}

Reference

1.14 (1.12 to 1.17 )

Reference

Reference

1.35 (1.23 to 1.49$)$

1.41 (1.19 to 1.66$)$

$1.17(0.84$ to 1.61$)$

$1.52(1.30$ to 1.79$)$

1.69 (1.33 to 2.16$)$

1.80 (1.54 to 2.10$)$

1.17 (0.87 to 1.57$)$

$1.23(1.10$ to 1.37$)$

0.93 (0.91 to 0.95)

Reference

1.40 (1.25 to 1.56$)$

$1.13(0.97$ to 1.31$)$

1.58 (1.26 to 1.98$)$

1.41 (1.17 to 1.71$)$

1.15 (0.90 to 1.48$)$

1.35 (1.16 to 1.58$)$

1.22 (0.94 to 1.58$)$

1.13 (1.00 to 1.29$)$

Reference

0.96 (0.94 to 0.98)

1.14 (1.09 to 1.19$)$

1.20 (1.16 to 1.24$)$

1.27 (1.21 to 1.33 )

Reference

1.04 (1.02 to 1.06$)$

1.11 (1.06 to 1.16$)$

1.04 (1.01 to 1.08)

1.09 (1.04 to 1.14)

1.02 (0.96 to 1.08$)$

1.08 (0.98 to 1.18$)$

1.22 (1.09 to 1.36$)$

1.22 (1.18 to 1.27$)$

1.05 (1.03 to 1.08$)$

1.05 (1.01 to 1.09)

$0.94(0.92$ to 0.96$)$

0.97 (0.94 to 1.01)

1.17 (1.08 to 1.26$)$

0.97 (0.95 to 1.00$)$

0.71 (0.61 to 0.82$)$

1.22 (1.08 to 1.39$)$

1.03 (0.97 to 1.10$)$

0.90 (0.81 to 0.99$)$

$0.93(0.82$ to 1.07$)$

1.03 (1.00 to 1.08$)$

0.96 (0.93 to 0.99)

0.94 (0.90 to 0.98)

1.01 (0.99 to 1.03$)$

0.97 (0.93 to 1.01)

1.02 (0.93 to 1.10$)$

1.06 (1.02 to 1.09$)$

0.75 (0.65 to 0.86$)$

1.00 (0.96 to 1.04)

1.74 (1.24 to 2.45$)$

1.02 (0.97 to 1.06$)$

0.83 (0.54 to 1.29)

0.83 (0.82 to 0.85$)$

0.89 (0.86 to 0.93$)$

1.05 (1.02 to 1.07$)$

0.97 (0.93 to 1.02)

1.00 (0.99 to 1.00$)$

1.03 (1.03 to 1.04 )

Reference

0.99 (0.94 to 1.03)

$1.00(0.95$ to 1.05$)$

1.05 (1.00 to 1.10$)$

1.06 (1.00 to 1.12$)$

Reference

0.91 (0.88 to 0.93 )

0.92 (0.87 to 0.97$)$

0.77 (0.72 to 0.82$)$

0.92 (0.81 to 1.04$)$

0.88 (0.85 to 0.91$)$

Reference

1.12 (1.10 to 1.15$)$

$0.60(0.57$ to 0.64$)$

All adjusted models include sex, ethnicity, smoking status, chronic conditions, use of other drugs, practice based Townsend score groups, and year of entering the study.

*Model also includes type and dose of statin at baseline, fractional polynomials for age ((age/5) and (age/5)2), body mass index (BMI; In(BMI), BMI0.5), total cholesterol:high density lipoprotein ratio (ratio 0.5 ), and systolic blood pressure (SBP; (SBP/10)-0.5).

tModel also includes type and dose of statin at discontinuation, fractional polynomials for age ((age/5)-1, (age/5)3), body mass index (BMI), total cholesterol:high density lipoprotein ratio (ratio-0.5), and systolic blood pressure ((SBP/10)). Practice ID was included as clustering variable.

¥The analysis was stratified by dementia. Results for discontinuation were taken from additional analyses by terms of use (supplementary table 3).

For all models, Practice ID was included as clustering variable.

§Estimates of Townsend score groups (patient based) are from the additional analysis (supplementary table 4). 

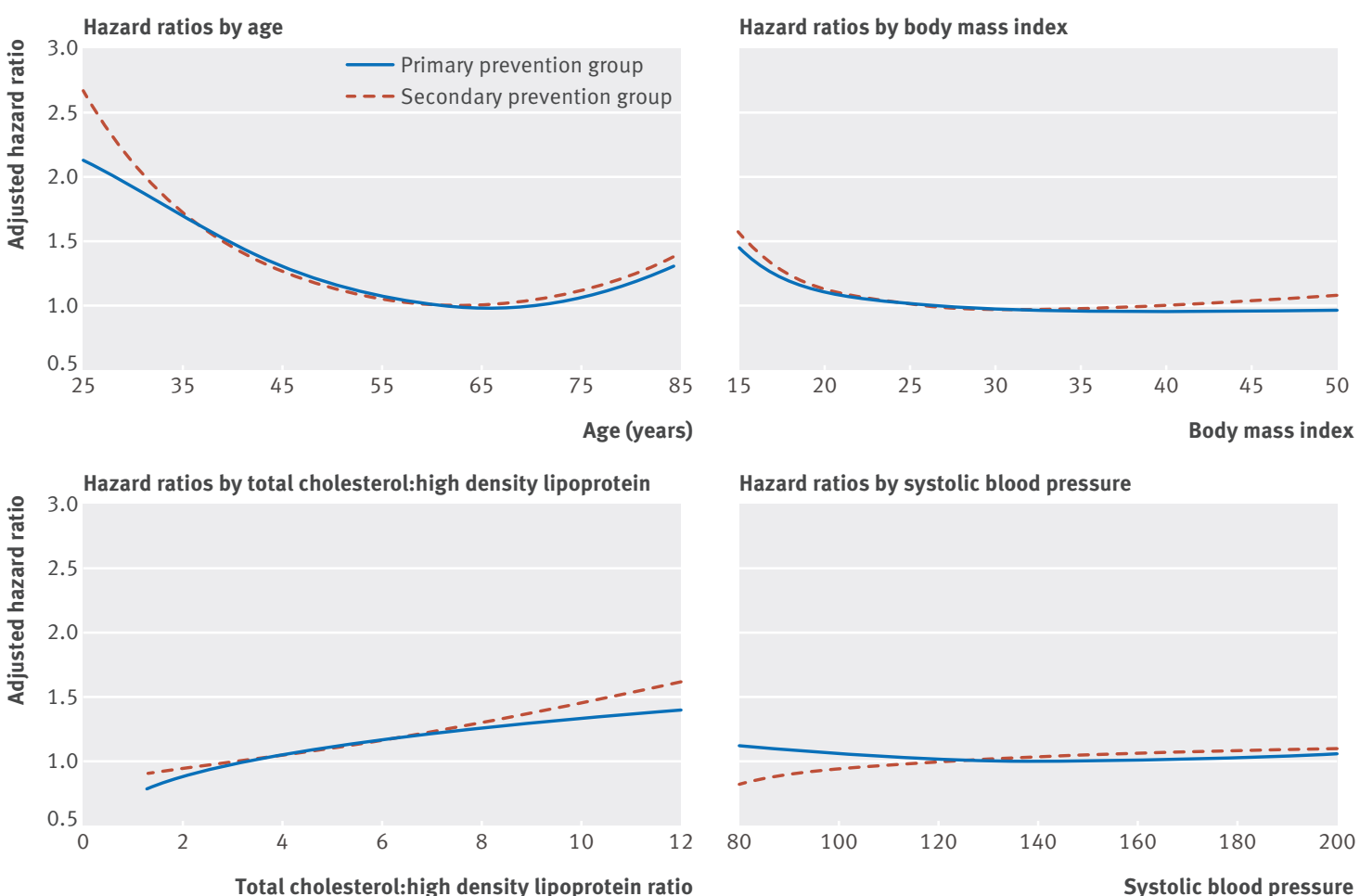

Hazard ratios by systolic blood pressure

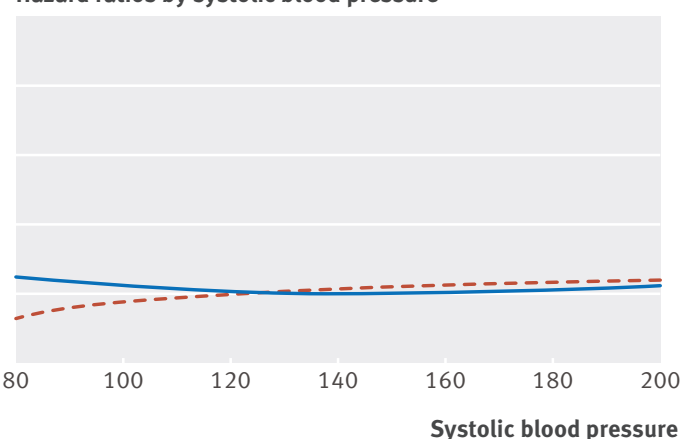

Fig 4 | Risk of discontinuation of statin treatment, as shown by fractional polynomials terms. Terms are for age (hazard ratios compared with age 60 years), body mass index (hazard ratios compared with body mass index 25), high density lipoprotein (HDL):total cholesterol ratio (hazard ratios compared with ratio 3.5), and systolic blood pressure (hazard ratios compared with $130 \mathrm{~mm} \mathrm{Hg}$ )
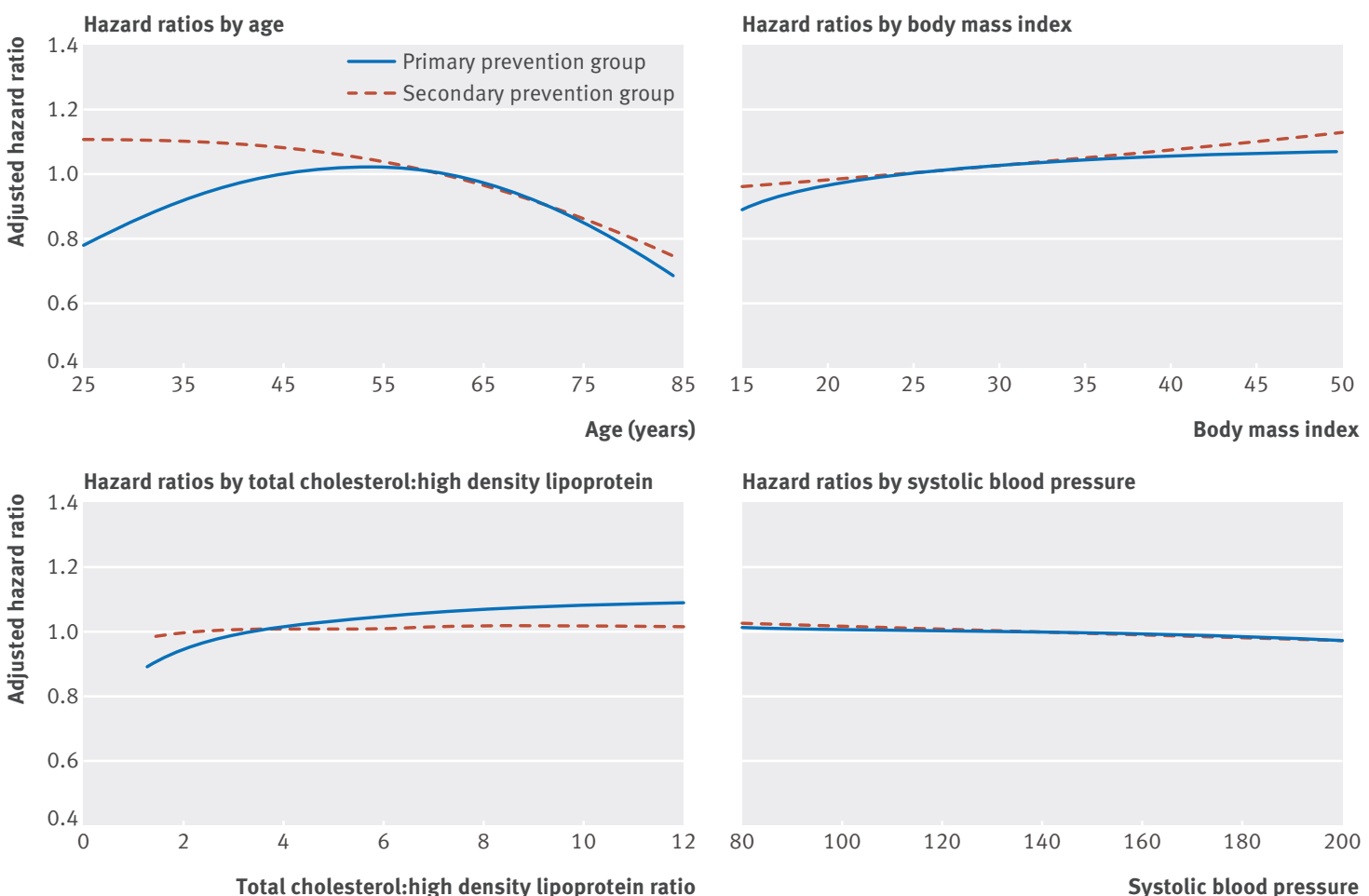

Hazard ratios by systolic blood pressure

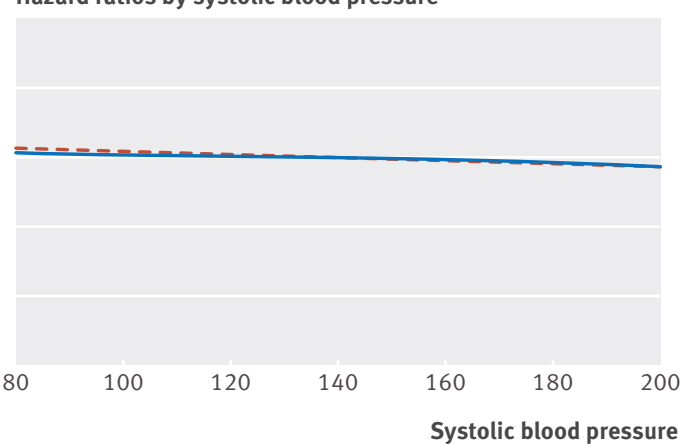

Fig 5 | Risk of restarting statin treatment, as shown by fractional polynomials terms. Terms are for age (hazard ratios compared with age 60 years), body mass index (hazard ratios compared with body mass index 25), high density lipoprotein (HDL):total cholesterol ratio (compared with ratio 3.5), and systolic blood pressure (compared with $130 \mathrm{~mm} \mathrm{Hg}$ ) 


\begin{tabular}{|c|c|c|}
\hline Risk factors & Discontinuation statin treatment & Restarting statin treatment \\
\hline \multicolumn{3}{|l|}{ Primary prevention group } \\
\hline \multicolumn{3}{|l|}{ Patients more likely to discontinue and less likely to restart } \\
\hline Women $v$ men & $+6 \%$ & $-8 \%$ \\
\hline Age from 25 to 50 years $v 60$ years & +111 to $+16 \%$ & $-23 \%$ to $0 \%$ \\
\hline Age from 75 to 84 years $v 60$ years & $+4 \%$ to $+29 \%$ & $-16 \%$ to $-32 \%$ \\
\hline Body mass index from 15 to $23 v 25$ & $+44 \%$ to $+3 \%$ & $-12 \%$ to $-1 \%$ \\
\hline Liver disease & $+17 \%$ & - \\
\hline Cancer & - & $-3 \%$ \\
\hline Dementia (long term statin use) & $+14 \%$ & $-20 \%$ \\
\hline Aspirin use & $+2 \%$ & $-8 \%$ \\
\hline Each other non-cardiovascular treatment & $+2 \%$ & - \\
\hline \multicolumn{3}{|l|}{ Patients more likely to discontinue and more likely to restart } \\
\hline Ethnic minority groups $v$ white & $+25 \%$ to $+89 \%$ & $+21 \%$ to $+46 \%$ \\
\hline Current smoking, from light to heavy $v$ non-smoking & $+7 \%$ to $+16 \%$ & $+5 \%$ to $+9 \%$ \\
\hline Chronic obstructive pulmonary disease & $+4 \%$ & $+6 \%$ \\
\hline Type 1 diabetes & $+18 \%$ & $+15 \%$ \\
\hline \multicolumn{3}{|l|}{ Patients less likely to discontinue or more likely to restart } \\
\hline Ex-smoking $v$ non-smoking & $-3 \%$ & - \\
\hline Atrial fibrillation & $-4 \%$ & - \\
\hline Hypertension & $-20 \%$ & $+8 \%$ \\
\hline Heart failure & $-8 \%$ & - \\
\hline Type 2 diabetes & $-20 \%$ & $+35 \%$ \\
\hline Family history of heart disease & - & $+4 \%$ \\
\hline Familial hypercholesterolaemia & $-14 \%$ & $+23 \%$ \\
\hline Anticoagulant use & $-12 \%$ & $-6 \%$ \\
\hline \multicolumn{3}{|l|}{ Secondary prevention group } \\
\hline \multicolumn{3}{|l|}{ Patients more likely to discontinue and less likely to restart } \\
\hline Women $v$ men & $+14 \%$ & $-7 \%$ \\
\hline Age from 75 to 84 years $v 60$ years & $+10 \%$ to $+35 \%$ & $-14 \%$ to $-26 \%$ \\
\hline Body mass index from 15 to $23 v 25$ & $+55 \%$ to $+3 \%$ & - \\
\hline Liver disease & $+22 \%$ & - \\
\hline Chronic obstructive pulmonary disease & $+22 \%$ & - \\
\hline Cancer & $+5 \%$ & $-4 \%$ \\
\hline Atrial fibrillation & $+5 \%$ & $-6 \%$ \\
\hline Type 1 diabetes & $+17 \%$ & - \\
\hline Dementia (long term use) & $+22 \%$ & - \\
\hline Familial hypercholesterolaemia & $+74 \%$ & - \\
\hline Each other non-cardiovascular treatment & $+3 \%$ & - \\
\hline \multicolumn{3}{|l|}{ Patients more likely to discontinue and more likely to restart } \\
\hline Age from 25 to $50 \mathrm{v} 60$ years & $+166 \%$ to $+11 \%$ & $+10 \%$ to $+5 \%$ \\
\hline Ethnic minority groups $v$ white & $+23 \%$ to $+80 \%$ & $+35 \%$ to $+58 \%$ \\
\hline Current smoking, from light to heavy $v$ non-smoking & $+14 \%$ to $+27 \%$ & $+4 \%$ to $+11 \%$ \\
\hline \multicolumn{3}{|l|}{ Patients less likely to discontinue or more likely to restart } \\
\hline Ex-smoking $v$ non-smoking & $-4 \%$ & $+4 \%$ \\
\hline Hypertension & $-6 \%$ & - \\
\hline Type 2 diabetes & - & $+6 \%$ \\
\hline Aspirin use & $-17 \%$ & $+5 \%$ \\
\hline Anticoagulant use & $-11 \%$ & - \\
\hline
\end{tabular}

commonly been reported, but our findings suggest that most patients often described as "stoppers" might more accurately be described as "non-adherent users." This study identified three broad categories of statin users within both primary and secondary prevention groups: patients who are likely to discontinue and not likely to restart (real stoppers), patients who are likely to discontinue but likely to restart (stop-starters), and patients who are less likely to discontinue or more likely to restart (relative adherents).

\section{Real stoppers}

In the first category of real stoppers, factors occurring in both prevention groups included women, older age, lower body mass index scores, chronic liver disease and multiple drugs. With respect to older patients, a review has noted that other conditions in the older population might affect statin metabolism or cause similar side effects, thus changing the statin pharmacokinetics for this group. ${ }^{27}$ In particular, muscular side effects have been shown elsewhere to be important in older patients treated with statins. ${ }^{28}$ The association between liver 
disease and increased risk of discontinuation followed by decreased risk of restarting could be related to statins causing an elevation of liver enzymes, which might cause doctors to stop prescribing the drug. ${ }^{29}$ The association between increased risk of discontinuation and the number of other treatments used apart from those related to cardiovascular treatment might result from patients having a more serious condition according lower status to preventive medications. Such patients also could be affected by the number of drug treatments or have side effects from interactions between drugs.

For the secondary prevention group only, patients with chronic obstructive pulmonary disease and type 1 diabetes also seemed more likely to be real stoppers. The finding for chronic obstructive pulmonary disease seems to have no obvious biological cause, conflicting with current biomedical research that suggests statins are beneficial to such patients, ${ }^{30}$ and a recent study showed decreased risk of exacerbations in statin users with the disease. ${ }^{31}$ Our findings suggest that there are features of having chronic obstructive pulmonary disease that make it difficult to adhere to statin treatment, which need further examination.

Increased risk of discontinuation in patients with type 1 diabetes has not been observed in previous studies, but a recent cross-sectional study on patients with type 1 diabetes ${ }^{32}$ showed an association between statin use and impaired glycaemic control. Although that study suggested revisiting insulin doses, in real settings this might lead to a higher level of statin discontinuation, and the increased risk of restarting statin treatment found in the primary prevention group might also suggest that this class of patients might be reintroduced to statins after a precautionary break.

Some factors relate to more personal circumstances. For example, relatively healthier patients (younger or with lower body mass index) in the primary prevention group might prefer to use other methods of lowering cardiovascular risk, such as aspirin use or exercise. On the other hand, more frail patients (older or those with serious comorbidities) might experience more problematic side effects or drug interactions. Patients with dementia could have particular problems in terms of treatment decisions and maintenance as their condition worsens.

\section{Stop-starters}

Factors in this category notably differed from the real stopper category, consisting primarily of patients with cultural or lifestyle risk factors-people from ethnic minorities and current smokers. Only two disease factors were included in this category, both in the primary prevention group. For chronic obstructive pulmonary disease, the increases in discontinuation and decreases in restarting were quite low and might reflect under-reporting in smoking status. For type 1 diabetes, where disturbances in glycaemic control might cause occasional adherence problems, the higher restarting risk might be partly explained by regular consultations to review glycated haemoglobin levels every three to six months at which statin use might also be discussed. For younger patients in the secondary prevention group, the stop-start tendency might be explained similarly to real stopping for this group in the primary group (but modified by experience of a past cardiovascular event).

As a lifestyle factor, current smoking is not surprising in this category because the dangers of this behaviour are well known and it is likely to signal either a general lack of concern for health issues or personal issues that have overwhelmed health concerns. The more complex result is the inclusion in this category across both prevention groups of ethnic minority status, albeit with different levels of outcome across different ethnicities. This could reflect a range of causes, which might-in different mixes and levels in different ethnicities-be more prevalent in some groups in the ethnic communities. Such factors include communication issues, cultural issues including attitudes to conventional medicine, and lifestyle issues. ${ }^{33} 34$

\section{Relative adherents}

Factors in this category mostly relate to cardiovascular conditions and the tendency to adherence is more pronounced in the primary prevention group. These factors could relate to a group of individuals at serious risk of cardiovascular events, who are concerned to avoid such events, particularly relatively younger or healthier patients in the primary prevention group. Two factors appear in both prevention groups: hypertension and use of anticoagulant drugs. In the primary prevention group only, patients with type 2 diabetes or familial hypercholesterolaemia showed similar lower risks of discontinuation. In the secondary prevention group, aspirin users also had lower risks of discontinuing. For both aspirin and anticoagulants, the lower risk of discontinuation in the secondary prevention group could point to increased treatment management for people being treated for serious cardiovascular problems.

\section{Other findings}

In general, our study found few associations between the type of statin used and poor adherence or stopping. We found no difference between the drug type and discontinuation risk in the primary group, and a $19 \%$ increased risk for rosuvastatin in the secondary group compared with simvastatin. The prescribed dose could reflect the severity of the existing cardiovascular disease or higher cardiovascular risk score, so patients on smaller doses were more likely to be real stoppers whereas patients on higher ones were more likely to be adherent.

\section{Comparisons with other studies}

With respect to statin adherence, a review has identified three groups of predictors: patient factors, physician factors, and healthcare system factors. ${ }^{35}$ But within the environment of freely available national healthcare in the UK, the last of these predictor groups is still largely irrelevant. The data source we used has no information on physician behaviour but, given the size and focus of our study, differences arising from patient characteristics 
are likely to overwhelm effects arising from doctor related issues. This study relates to patient issues, which is the most complex aspect, potentially spanning physical and psychological personal characteristics, genetic and other health effects, cultural issues, and lifestyle choices. Our data source, however, does not allow us to go further than examine discontinuation and restarting rates associated with some of these factors or proxies for them.

\section{Discontinuation rate}

In earlier studies, definitions of both discontinuation and non-adherence have differed widely, creating a challenge for several meta-analyses. ${ }^{151636}$ One of these meta-analyses included both randomised controlled trials and observational studies, ${ }^{15}$ showing that adherence levels at one year reported in randomised controlled trials were on average much higher $(90.3 \%$ (95\% confidence interval $89.9 \%$ to $90.8 \%$ )) than those reported in observational studies (49\% (48.9\% to $49.2 \%)$ ). In our study, $75 \%$ of patients were still on statins by the end of the first year.

Further comparisons are difficult because observational studies have not been consistent in their design, with definition of discontinuation varying from 30 days $^{37}$ to 12 months. ${ }^{19}$ Studies using reimbursement or dispensing data suggest lower adherence estimates than studies based on prescribing data because patients who receive prescriptions might not fill these at a pharmacy. Several other minor differences between our study and others probably reflect data selection issues such as patient age range and data source (general population, insurance data, surveys, or hospital discharges).

\section{Discontinuation risk factors}

A meta-analysis based on observational studies used pharmacy and insurance database refills, and included only studies with validated adherence measures. ${ }^{16}$ It was designed to identify reliable predictors of non-adherence to statins, combining results from studies with different sets of available variables and different definitions of non-adherence. This study did not distinguish between primary and secondary prevention, but diagnosis of cardiovascular disease, younger and older age, female sex, and lower income were selected as factors in this study, and our results broadly agree with these and other outcomes. Regimen complexity, the relation between an increasing number of non-cardiovascular treatments and low adherence, gave similar results to our study, but the better adherence association with hypertension and with diabetes (type 2 only in our study) was found only in our primary prevention group.

Later observational studies were consistent with our results in identifying discontinuation factors such as diagnosis of cardiovascular disease, ${ }^{38}$ female sex, ${ }^{33} 38$ younger and older age, ${ }^{26}$ smoking, ${ }^{34}$ and normal weight. ${ }^{39}$ Our findings of increased discontinuation risks for ethnic minorities agree with results from another Danish study ${ }^{33}$ and an Australian study, ${ }^{34}$ which reported increased risks in patients using a language other than English at home.

\section{Restarting}

Most of the existing studies looked only at factors associated with discontinuation, and only a few investigated the process of restarting. In a Canadian study, $54 \%$ of statin users discontinued the treatment but $57 \%$ of these restarted. ${ }^{17}$ In our study, despite the longer study period, the proportion of discontinuers was slightly lower (46\%) and the proportion of restarters was higher (73\%). The difference might be explained by timing, because the Canadian study was conducted on data between 1997 and 2004, when there was less evidence to support the use of statins. The study also concentrated on adverse events or visits to physicians as possible factors for restarting and did not investigate any patient level characteristics by incorporating them in the design.

Another study in the USA, similar to ours, considered restarting and showed that younger age and female sex were associated both with increased risk of discontinuation and decreased risk of restarting. ${ }^{18}$ Zhang and colleagues ${ }^{19}$ investigated the reasons for discontinuation among 57292 patients of an original cohort of 107835 statin users. The proportion of patients who discontinued was 53\%, which was much higher than in our study despite their longer 12 month gap for discontinuation, while the proportion of those restarting was 64\%, which was similar to our study. Unlike our study, Zhang and colleagues did not attempt to identify groups of patients more likely to discontinue or to restart.

\section{Strengths and limitations}

This large study was based on a representative sample of statin users from the general population. The size and rigorous design has facilitated investigation of several factors identified in previous studies and meta-analyses within a single coherent structure. With separate information for the primary and secondary prevention groups, and a long period of follow-up, the study delivers valuable information about various issues, in particular the less studied aspect of discontinuation and restarting rates, which gives a more comprehensive overview of statin adherence.

There are several limitations related to our sources of information. Information on statin use was limited to estimates based on prescription data, so actual statin use might be overestimated because some patients might not fill the prescription in the pharmacy or, having received pills, actually take them. Patients with one prescription only were excluded from the main analysis, but it is possible that they started treatment and discontinued during or after the first prescription. Exclusion of these patients could have led to a slightly underestimated rate of discontinuation. The definition of discontinuation as a 90 day gap might also not capture patients who discontinued for less than 90 days and then restarted.

Reasons for discontinuation could not be included because, if recorded, this is usually in the form of free 
text, which was not available to this study. Coded information relating to reasons was available only for a few patients $(<1 \%)$. Not all factors previously identified as possibly relevant to discontinuation were available. For example, frequent exercise, for which we lacked information, is a way of decreasing cardiovascular risk and could have a double effect on discontinuation-improved health or increased risk of statin side effects such as myalgia. ${ }^{40}$ However, a Finnish survey ( $\mathrm{n}=9285)$ did include physical activity as a possible predictor, but found no significant association with statin non-adherence in either primary or secondary prevention groups. ${ }^{39}$ Another study, which identified smoking as a predictor of non-adherence (as we did), also identified being employed, higher level of education, and psychological distress, which we could not investigate. ${ }^{34}$

An important limitation was lack of information about patient attitudes, especially with respect to preventive treatment for the primary group..$^{41}$ A survey conducted on patients who did not fill their new statin prescription has shown that the most common reasons for this were the decision to change their lifestyle (63\%) and a fear of side effects (53\%). ${ }^{42}$ We also had no information about physician characteristics or adherence to guidelines, or about contacts between doctor and patient.

\section{Implications}

Because of the lack of data on reasons for discontinuations, this study cannot directly determine why patients discontinue statin treatments. What it does do with some certainty is identify groups of patients where a shared risk factor is associated with increased or decreased levels of discontinuation or restarting compared with their reference group.

Although the risk factors in each broad category differ in detail between the primary and secondary prevention groups, there is a high degree of commonality. Some differences also clearly reflect the varying circumstances faced by patients who are at risk of a cardiovascular event compared with those who have actually had one. All categories include useful information for doctors and patients, and for researchers interested in more detailed and focused studies using more complete or exact data sources.

The first two categories of statin users-real stoppers and stop-starters-are potentially of greatest interest and use. Across both, doctors and patients could make use of information from this study. Doctors could use the information to identify which patients might need more help and encouragement to maintain adherence. Our results could also highlight to patients that many people like them or with their condition have tended to show real stopping or stop-starting adherence patterns. Patients might then be encouraged to discuss such issues with their doctor to try to discover solutions, alternatives, or compromises that could improve their adherence.

Researchers might also be interested in the outcomes from either of these categories-particularly in those which are least easily explained or where non-adherence for a patient might carry most risks. In terms of stop-starting tendencies in some members of ethnic communities, this evidence suggests several research questions that either apply directly to adherence to statins or other drugs, or as part of wider studies looking at ways of spreading the potential benefits of NHS healthcare to sections of the population who are more difficult to reach.

\section{Conclusion}

We have found that, although more than $40 \%$ of statin users do discontinue their treatment at some point, more than $70 \%$ of the discontinuers restart. We have also identified those patient factors linked to discontinuation and restarting patterns, which suggest relative adherence, real stopping, or stop-starting behaviour. Our overall levels of restarting suggest that, with a few exceptions, the problem of statin stopping could be part of the wider issue of temporary stopping or poor adherence.

We have also identified those groups of patients, such as women, who might be less likely to restart once discontinued, which has the potential to facilitate greater focus by clinicians on these groups. Information on groups with high levels of both discontinuation and restarting, such as patients from ethnic minority groups, might also encourage better understanding by clinicians of the issues. Findings relating to unexpected discontinuation or adherence, such for patients with chronic obstructive pulmonary disease, deserve further study. With respect to ethnic minorities, our results suggest the need for further qualitative research based on access to a broader range of detailed social and cultural information to clarify the reasons for this important outcome.

Contributors: $\mathrm{JH}-\mathrm{C}$ had the original idea for this study. $\mathrm{JH}-\mathrm{C}, \mathrm{CC}$, and PB contributed to the development of the idea and the study design. $\mathrm{YV}$ reviewed the literature, contributed to the study design, did the analysis, and drafted the manuscript. CC advised on the analysis. JH-C, $\mathrm{PB}$, and CC critically reviewed the paper. $\mathrm{YV}$ is the guarantor of the study. All authors have approved the submitted version.

Funding: This work has received no specific grant from any funding agency in the public, commercial, or not-for-profit sectors. Only the authors are responsible for analysis, interpretation of the data, and writing the report for publication.

Competing interests: All authors have completed the ICMJE uniform disclosure form at www.icmje.org/coi_disclosure.pdf and declare: no support from any additional organisation for the submitted work; JH-C is a professor of clinical epidemiology at the University of Nottingham and unpaid director of QResearch, a not-for-profit organisation that is a joint partnership between the University of Nottingham and EMIS (Egton Medical Information System; commercial IT supplier for $60 \%$ of general practices in the UK); JH-C is also a paid director of ClinRisk, which produces open and closed source software to ensure the reliable and updatable implementation of clinical risk algorithms (including QRISK2) within clinical computer systems to help improve patient care; no other relationships or activities that could appear to have influenced the submitted work.

Ethical approval: This protocol has been approved by the independent scientific advisory committee for Medicines and Healthcare products Regulatory Agency Database Research (N 15_053RAR2). The protocol has been published in BMJ Open. ${ }^{20}$

Data sharing: To guarantee the confidentiality of personal and health information, only the authors have had access to the data during the study. It will be possible to access the CPRD data after the publication of the results, but only on premises of the University of Nottingham according to the CPRD licence. 
The lead author affirms that the manuscript is an honest, accurate, and transparent account of the study being reported; that no important aspects of the study have been omitted; and that any discrepancies from the study as planned (and, if relevant, registered) have been explained.

This is an Open Access article distributed in accordance with the Creative Commons Attribution Non Commercial (CC BY-NC 3.0) license, which permits others to distribute, remix, adapt, build upon this work non-commercially, and license their derivative works on different terms, provided the original work is properly cited and the use is non-commercial. See: http://creativecommons.org/licenses/

by-nc/3.0/

1 Trusler D. Statin prescriptions in UK now total a million each week. BM/ 2011;343:d4350. doi:10.1136/bmi.d4350.

2 Stone NJ, Robinson JG, Lichtenstein AH, et al. American College of Cardiology/American Heart Association Task Force on Practice Guidelines. 2013 ACC/AHA guideline on the treatment of blood cholesterol to reduce atherosclerotic cardiovascular risk in adults: a report of the American College of Cardiology/American Heart Association Task Force on Practice Guidelines [corrections in: / Am Coll Cardiol 2015;66:2812 and J Am Coll Cardiol 2014;63:3024-5]. J Am Coll Cardiol 2014;63(25, Part B):2889-934. doi:10.1016/j. jacc.2013.11.002

3 National Institute for Health and Care Excellence. Lipid modification cardiovascular risk assessment and the modification of blood lipids for the primary and secondary prevention of cardiovascular disease. Clinical quideline 181. NICE, 2014.

4 National Institute for Health and Care Excellence. Lipid modification. cardiovascular risk assessment and the modification of blood lipids for the primary and secondary prevention of cardiovascular disease. Clinical guideline 67. NICE, 2008.

5 Mihaylova B, Emberson J, Blackwell L, et al. Cholesterol Treatment Trialists' (CTT) Collaborators. The effects of lowering LDL cholesterol with statin therapy in people at low risk of vascular disease: meta-analysis of individual data from 27 randomised trials. Lancet 2012;380:581-90. doi:10.1016/S0140-6736(12)60367-5.

6 Taylor F, Huffman MD, Macedo AF, et al. Statins for the primary prevention of cardiovascular disease. Cochrane Database Syst Rev 2013;1:CD004816

7 Abramson JD, Rosenberg HG, Jewell N, Wright JM. Should people at low risk of cardiovascular disease take a statin [correction in: BM] 2014;348:g3329]? BMJ 2013;347:f6123. doi:10.1136/bmj.f6123.

8 Malhotra A. Saturated fat is not the major issue [correction in: $B M$. 2014:348:53332]. BM/2013:347:f6340, doi:10.1136/bmi.f6340.

9 Prasad V. Statins, primary prevention, and overall mortality. Ann Intern Med 2014;160:867-9. doi:10.7326/M13-2974.

10 Hippisley-Cox J, Parker C, Coupland C, Vinogradova Y. Inequalities in the primary care of patients with coronary heart disease and serious mental health problems: a cross-sectional study. Heart 2007;93:125662. doi:10.1136/hrt.2006.110171

11 Hippisley-CoxJ, Pringle M, Crown N, Meal A, Wynn A. Sex inequalities in ischaemic heart disease in general practice: cross sectional survey. BMJ 2001;322:832. doi:10.1136/bmj.322.7290.832.

12 Hippisley-Cox J, Coupland C. Unintended effects of statins in men and women in England and Wales: population based cohort study using the QResearch database. BMJ 2010;340:c2197. doi:10.1136/bmj. C2197.

13 Sattar N, Preiss D, Murray HM, et al. Statins and risk of incident diabetes: a collaborative meta-analysis of randomised statin trials. Lancet 2010;375:735-42. doi:10.1016/S0140-6736(09)61965-6.

14 Smeeth L, Hubbard R, Fletcher AE. Cataract and the use of statins: a case-control study. Q/M 2003:96:337-43. doi:10.1093/qimed/ hcg064.

15 Lemstra M, Blackburn D, Crawley A, Fung R. Proportion and risk indicators of nonadherence to statin therapy: a meta-analysis. Can 」 Cardiol 2012;28:574-80. doi:10.1016/i.cjca.2012.05.007.

16 Mann DM, Woodard M, Muntner P, Falzon L, Kronish I. Predictors of non-adherence to statins: a systematic review and meta-analysis. Annals Pharmacother 2010:44:1410-21.

17 Brookhart MA, Patrick AR, Schneeweiss S, et al. Physician follow-up and provider continuity are associated with long-term medication adherence: a study of the dynamics of statin use. Arch Intern Med 2007;167:847-52. doi:10.1001/archinte.167.8.847.

18 Caspard H, Chan AK, Walker AM. Compliance with a statin treatment in a usual-care setting: retrospective database analysis over 3 years after treatment initiation in health maintenance organization enrollees with dyslipidemia. Clin Ther 2005;27:1639-46. doi:10.1016/j.clinthera.2005.10.005

19 Zhang H, Plutzky J, Skentzos S, et al. Discontinuation of statins in routine care settings: a cohort study. Ann Intern Med 2013;158:52634. doi:10.7326/0003-4819-158-7-201304020-00004
20 Vinogradova Y, Coupland C, Brindle P, Hippisley-Cox J. Patients who discontinued statin treatment: a protocol for cohort study using primary care data. BMJ Open 2015:5:e008701. doi:10.1136/ bmjopen-2015-008701.

21 Daskalopoulou SS, Delaney JAC, Filion KB, Brophy JM, Mayo NE, Suissa S. Discontinuation of statin therapy following an acute myocardial infarction: a population-based study. Eur Heart / 2008;29:2083-91. doi:10.1093/eurheartj/ehn346.

22 Jones PH, Davidson MH, Stein EA, et al. STELLAR Study Group. Comparison of the efficacy and safety of rosuvastatin versus atorvastatin, simvastatin, and pravastatin across doses (STELLAR* Trial). Am / Cardiol 2003;92:152-60. doi:10.1016/ S0002-9149(03)00530-7.

23 Royal Pharmaceutical Society of Great Britain, British Medical Association. British National Formulary 66. Pharmaceutical Press, 2013.

24 Royston P. Multiple imputation of missing values. Stata I 2004:4:227-41.

25 Steyerberg EW, van Veen M. Imputation is beneficial for handling missing data in predictive models. J Clin Epidemiol 2007;60:979. doi:10.1016/j.jclinepi.2007.03.003.

26 Svensson E, Nielsen RB, Hasvold P, Aarskog P, Thomsen RW. Statin prescription patterns, adherence, and attainment of cholesterol treatment goals in routine clinical care: a Danish population-based study. Clin Epidemiol 2015;7:213-23.

27 Casula M, Tragni E, Catapano AL. Adherence to lipid-lowering treatment: the patient perspective.Patient prefer adherence 2012:6:805-14.

28 Rosenbaum D, Dallongeville J, Sabouret P, Bruckert E. Discontinuation of statin therapy due to muscular side effects: a survey in real life. Nutr Metab Cardiovasc Dis 2013;23:871-5. doi:10.1016/i. numecd 2012.04.012.

29 Pastori D, Polimeni L, Baratta F, Pani A, Del Ben M, Angelico F. The efficacy and safety of statins for the treatment of non-alcoholic fatty liver disease. Dig Liver Dis 2015;47:4-11. doi:10.1016/j. dld.2014.07.170.

30 Young RP, Hopkins R, Eaton TE. Pharmacological actions of statins: potential utility in COPD. Eur Respir Rev 2009:18:222-32. doi:10.1183/09059180.00005309.

31 Ingebrigtsen TS, Marott JL, Nordestgaard BG, Lange P, Hallas J, Vestbo 1. Statin use and exacerbations in individuals with chronic obstructive pulmonary disease. Thorax 2015;70:33-40. doi:10.1136/ thoraxjnl-2014-205795.

32 Jensen MT, Andersen HU, Rossing P, Jensen IS. Statins are independently associated with increased HbA1c in type 1 diabetes-The Thousand \& 1 Study. Diabetes Res Clin Pract 2016;111:51-7. doi:10.1016/j.diabres.2015.10.022.

33 Nielsen SF, Nordestgaard BG. Negative statin-related news stories decrease statin persistence and increase myocardial infarction and cardiovascular mortality: a nationwide prospective cohort study. Eur Heart/ 2016;37:908-16. doi:10.1093/eurhearti/ehv641.

34 Warren IR, Falster MO, Fox D, Jorm L. Factors influencing adherence in long-term use of statins. Pharmacoepidemiol Drug Saf 2013;22:1298307. doi:10.1002/pds.3526.

35 Mauskop A, Borden WB. Predictors of statin adherence. Curr Cardiol Rep 2011;13:553-8. doi:10.1007/s11886-011-0221-2.

36 Schedlbauer A, Davies P, Fahey T. Interventions to improve adherence to lipid lowering medication. Cochrane Database Syst Rev 2010:(3):CD004371.

37 Larsen J, Andersen M, Kragstrup J, Gram LF. High persistence of statin use in a Danish population: compliance study 1993-1998. Br J Clin Pharmacol 2002:53:375-8. doi:10.1046/i.1365-2125.2002.01563.x.

38 Ferrajolo C, Arcoraci V, Sullo M, et al. Pattern of statin use in southern italian primary care: long-term adherence to the treatment [correction in: PLoS One 2014;9:e112037]. PLoS 2014;9:e102146. doi:10.1371/ journal pone. 0102146

39 Halava H, Korhonen MJ, Huupponen R, et alLifestyle factors as predictors of nonadherence to statin therapy among patients with and without cardiovascular comorbidities. CMA/ 2014:186:E449-56.

40 Mancini GBJ, Tashakkor AY, Baker S, et al. Diagnosis, prevention, and management of statin adverse effects and intolerance: Canadian Working Group Consensus update. Can I Cardiol 2013;29:1553-68. doi:101016/j.cica 2013.09 .023$.

41 Granger BB, McBroom K, Bosworth HB, Hernandez A, Ekman I. The meanings associated with medicines in heart failure patients. Eurf Cardiovasc Nurs 2013:12:276-83. doi:10.1177/1474515112447734.

42 Harrison TN, Derose SF, Cheetham TC, et al. Primary nonadherence to statin therapy: patients' perceptions. Am J Manag Care 2013;19:e133-9.

Supplementary appendix: Additional tables 